\title{
Automated Estimation of Regional Mean Transition Times and Radial Velocities From Cine Magnetic Resonance Images: Evaluation in Normal Subjects
}

\author{
Racha El-Berbari, PhD, ${ }^{1,2}$ Nadjia Kachenoura, $\mathrm{PhD},{ }^{1}$ Alban Redheuil, MD, ${ }^{1,3}$ \\ Alain Giron, PhD, ${ }^{1}$ Elie Mousseaux, PhD, MD, ${ }^{1,3}$ Alain Herment, PhD, ${ }^{1}$ \\ Isabelle Bloch, $\mathrm{PhD},{ }^{2}$ and Frédérique Frouin, $\mathrm{PhD}^{1 *}$
}

\begin{abstract}
Purpose: To assess regional ventricular function via an accurate and automated definition of functional parameters.

Materials and Methods: An automated method is proposed that estimates reliable regional normalized mean transition times $\left(F_{m d}\right)$ and mean radial velocities $\left(V_{m}\right)$ from cine images. This approach combines a quantitative parametric imaging method and an automated detection of the endocardial border, which is robust to the presence of papillary muscles and nonhomogeneities within the left ventricular cavity. Steadystate free-precession cine-magnetic resonance imaging (MRI) of 36 healthy subjects was analyzed.
\end{abstract}

Results: The quality of the automated segmentation was assessed on a subgroup of 20 subjects by comparing the results with the manual contours traced by an expert. The comparison of functional parameters estimated consequently using the automated and the manual contours yielded $(y=0.959 x+0.016, R=0.964)$ for $F_{m c}$ and $(y=$ $0.883 x+0.505, R=0.935$ ) for $V_{m}$. On the entire group, $F_{m c}$ was equal to $0.392 \pm 0.069$ and $V_{m}$ to $5.4 \pm 2.3 \mathrm{~cm} / \mathrm{s}$. Increasing values of the temporal parameter from the apex to the base and larger values in the septal wall than in lateral wall were demonstrated and were in accordance with the physiology.

Conclusion: The proposed method ensures an automated and robust assessment of regional wall motion parameters, which could be clinically useful.

Key Words: cine MR imaging; regional wall motion analysis; mean transition time; radial endocardial velocity; quantitative parametric imaging; left ventricular segmentation

J. Magn. Reson. Imaging 2009;30:236-242.

(c) 2009 Wiley-Liss, Inc.
${ }^{1}$ INSERM, U678, UPMC Univ Paris 06, UMR_S 678, Paris, France. ${ }^{2}$ TELECOM Paris Tech (ENST), TSI Department, CNRS UMR 5141, Paris, France.

${ }^{3}$ AP-HP, Hôpital Européen Georges Pompidou, Department of Cardiovascular Radiology, Paris, France.

*Address reprint requests to: F.F., Laboratoire d'Imagerie Fonctionnelle, INSERM U678, 91 Boulevard de l'Hôpital, F-75634 Paris cedex 13, France. E-mail: frouin@imed.jussieu.fr

Received November 17, 2008; Accepted March 16, 2009.

DOI 10.1002/jmri.21798

Published online in Wiley InterScience (www.interscience.wiley.com).
THE EXCELLENT CONTRAST between the blood pool and the myocardium obtained using steady-state freeprecession cine sequence (SSFP) enables a better endocardial border definition and a lower interobserver variability of the assessment of the regional contraction compared to turbo gradient-echo sequences (1). Clinical assessment of the left ventricular (LV) segmental function is based mostly on visual interpretation. Accurate diagnosis requires the ability of the reader to integrate efficiently both temporal and spatial information on endocardial wall motion and myocardial wall thickening. To be reliable, such a visual analysis requires extensive training. Despite the high quality of cardiovascular magnetic resonance (CMR) images, the intra- and interoperator reproducibility in detecting regional wall motion abnormalities remains far from optimal (2).

To reduce the variability of visual wall motion analysis, quantitative postprocessing methods have been proposed on magnetic resonance images (MRI) (3-6). A parametric imaging method taking into account both wall excursion and chronological features in wall motion (7) was adapted to short-axis SSFP cine MRI in order to extract the segmental mean transition times, and radial endocardial velocities directly from cine MR images (8). A first evaluation has shown that mean transition times increased and mean radial velocities decreased in pathological segments when compared to normal segments. However, the estimation of these physiological parameters requires an accurate delineation of the endocardial LV border on the end-diastolic image, which was achieved manually. Accordingly, the main objective of the present study was to automate the detection of the endocardial border in order to increase the objectivity of the technique and its usefulness in a clinical setting.

A large number of automatic and semiautomatic procedures, based on various image processing approaches, have been proposed for LV segmentation (9-14). Among them, active contour models, or snakes, have received much interest. However, they converge toward a local minimum and thus depend on the initialization. To reduce this drawback, a new 
class of external forces called gradient vector flow (GVF), which is characterized by a non-null attraction even far from contours (15), has been used for LV delineation $(9,12)$. Despite this latter improvement, this approach still does not take into account flowrelated signal loss and lack of clear delineation between the myocardium and adjacent anatomic structures such as papillary muscles. Accordingly, the GVF snake approach was applied on images previously filtered using connected morphological operators (16).

This article describes the process, which combines the automated detection of the endocardium border with the parametric imaging-based quantification, and the evaluation protocol used for its validation. A subgroup of 20 subjects was used to assess the accuracy of the automated segmentation. This was achieved by comparing 1) the automated borders against those manually traced by an expert, and 2) the functional wall motion parameters, such as regional mean transition times and radial endocardial velocities, obtained using the automated contours and then the manual contours. Finally, the automated process was applied to the whole study group ( $n=36$ ), to estimate the functional parameters of time and velocity, and to define their normal values and their spatial variations.

\section{MATERIALS AND METHODS \\ Population and Image Acquisition}

After giving informed consent, 36 healthy subjects (28 men, 8 women, mean age $=34$ years, range $=$ 13-59 years) were screened for personal and family history of cardiovascular and pulmonary disease. They had no cardiovascular risk factor, no known acute or chronic disease, and no medication and all were asymptomatic and had a normal clinical examination (normal ECG, normal LV and RV function). All subjects underwent established techniques of cine CMR for function using 1.5-T MR equipment (Signa LX, GE Medical Systems, Milwaukee, WI) and 8-channel phased-array torso coil. Eight to 14 short-axis slices were acquired using an SSFP sequence: fast imaging employing steady-state acquisition (FIESTA). These slices were planned perpendicular to the axis joining the center of the mitral annulus and the apex defined on two orthogonal views (4-chamber and 2-chamber views) to include the entire left ventricular myocardium. The following acquisition parameters were used: repetition time: 3.7-4 msec; echo time: 1.6-1.7 msec; flip angle: $50^{\circ}$; view-persegment: 10-16; number of cardiac phases: 20-40 after view sharing; slice thickness: $8 \mathrm{~mm}$; interslice gap: $1 \mathrm{~mm}$; pixel size: $0.7 \times 0.7$ to $1.7 \times 1.7 \mathrm{~mm}^{2}$. The temporal resolution ranged between 20 and $40 \mathrm{msec}$ including 13 subjects between 20 and $25 \mathrm{msec}, 21$ subjects between 25 and $35 \mathrm{msec}$, and 2 subjects with 40 msec.

\section{Estimation of the Functional Parameters}

The whole process is summarized in Fig. 1.

\section{Slice Selection}

For each subject, an extreme basal slice in which the entire LV cavity was surrounded by the myocardium, and an extreme apical slice in which the LV cavity can be visualized during the entire cardiac cycle, were defined. Then all the slices, which are located between these two extreme slices, were selected for analysis. For each subject the included slices were visually assigned into three categories: 1) apical (AP) slices; 2) mid-ventricular or papillary muscles (PM) slices; and 3) basal or mitral valve (MV) slices.

\section{Area Selection}

For each slice, an operator defined two points manually on the end-diastolic image: $P_{0}$ in the center of the LV cavity, and $P_{1}$ the anterior attachment of the right ventricular wall to the LV. The field of view was reduced to a square region centered on $P_{0}$, the square side dimension was set to three times the distance between $P_{0}$ and $P_{1}$. These two points were used to automatically define six standardized segments [3]: anterior (A), anterolateral (AL), inferolateral (IL), inferior (I), inferoseptal (IS), and anteroseptal (AS) segments.

\section{Parametric Analysis of Main Motion}

This method (7) synthesizes the information contained in the cine images corresponding to one cardiac cycle into four parametric images related to the contraction amplitude and timing. It is based on the modeling of the time signal intensity curve $P(x, y, t)$ of each pixel $(x, y)$ using a window function:

$$
\begin{aligned}
& P(x, y, t)=A_{B}(x, y) \\
& \quad-A_{V}(x, y) \cdot g\left(t, T_{O N}(x, y), T_{O F F}(x, y)\right)+e(x, y, t) .
\end{aligned}
$$

The parameter $A_{B}(x, y)$ refers to the signal intensity at end-diastole, $T_{O N}(x, y)$ is the time at which the pixel $(\mathrm{x}, \mathrm{y})$ transitions from LV cavity into myocardium at the beginning of the contraction over $(x, y)$ implying a sharp decrease in signal intensity, $T_{O F F}(x, y)$ is the time at which the pixel $(\mathrm{x}, \mathrm{y})$ transitions from myocardium into LV cavity at the beginning of the LV relaxation over $(x, y)$ implying a sharp increase in signal intensity, and $A_{V}(x, y)$ is the variation of signal intensity caused by the transition of the myocardium over the pixel $(x, y)$ during the contraction phase (Fig. 1b). The function $g(t)$ is the window function and $e(x, y, t)$ is the error term. The four parameters were estimated for each individual pixel using a fast algorithm based on the least-square minimization of the modeling errors (7), and were then stored into four parametric images. An additional image of mean transition time, $T_{m c}(x, y)$, which is related to the chronology of the endocardial motion and in which higher values represent a delayed contraction was defined as:

$$
T_{m c}=\left(T_{O N}+T_{O F F}\right) / 2
$$

Finally, the trigger delay was added to the temporal parameters to set the zero of the measurements to the $\mathrm{R}$ wave. 

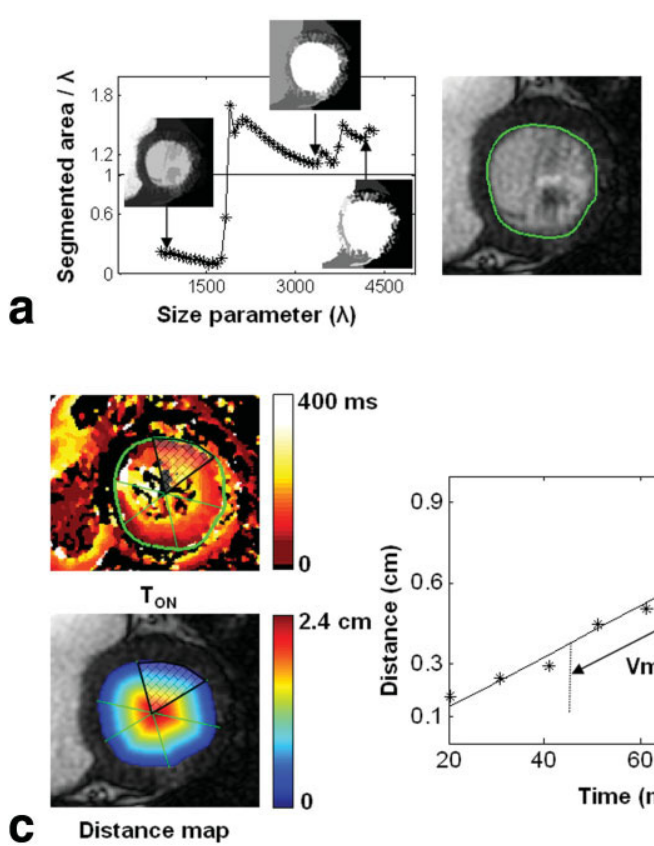

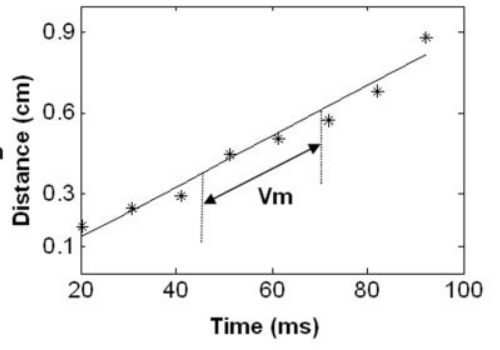

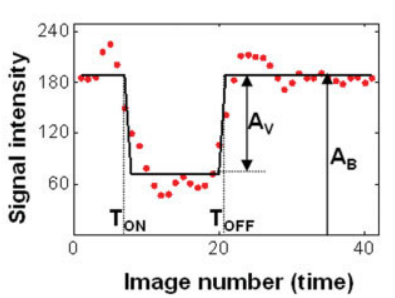

b End-systole

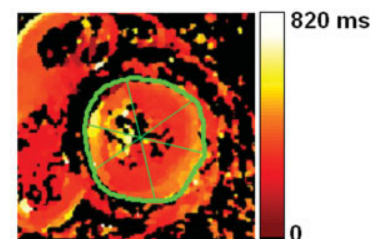

$T_{\mathrm{mc}}=\frac{T_{\text {ON }}+T_{\text {OFF }}}{2}$

Figure 1. Overview of the quantification process. a: Segmentation step. Left: effect of the size parameter $(\lambda)$ on the filtered images, with homogeneous LV cavity obtained for the optimal value of $\lambda$. Right: automated detection of the endocardial contour on the end-diastolic image, using the filtering process and the GVF snake algorithm. b: Principles of the parametric analysis of main motion and estimation of the two transition times $T_{O N}$ and $T_{O F F}$ by modeling the time signal intensity variation in a fixed pixel during a cardiac cycle with a window function. c: Quantification of the regional functional parameters. Left: estimation of mean regional radial velocity $\left(V_{m}\right)$ by combining the $T_{O N}$ parametric image with a distance map estimated from the end-diastolic endocardial contour. The linear regression of the dot plot extracted here from the anterior segment allows the estimation of $V_{m}$. Right: mean transition time image from which segmental values of $F_{m c}$ are extracted.

\section{Automated LV Segmentation}

The segmentation procedure was previously described (16), only its principles and its implementation are given here. The papillary muscles and the LV cavity were first merged using connected operators (area opening and area closing), resulting in a filtered image which mostly contains flat and large regions. For the area opening process, a size parameter $\lambda$ was defined such as the filtered image contains only connected components of area larger than $\lambda$ (17). A set of filtered images was obtained by varying the value of $\lambda$ and was segmented by GVF snakes (15), then the ratios between the area defined by the segmentation and the corresponding $\lambda$ were estimated. The value of $\lambda$ for which the ratio was the closest to 1 (Fig. 1a) was defined as the optimal value (16).

The GVF snake is a curve $X(s)=[x(s), y(s)], s \in[0,1]$ that evolves through the spatial domain of an image in order to achieve a balance between internal forces $F_{\text {int }}$ and external forces $F_{\text {ext }}$ :

$$
\begin{aligned}
F_{\text {int }}=\alpha X^{\prime \prime}(s)- & \beta X^{\prime \prime \prime \prime}(s) \\
& \text { and } F_{\text {ext }}=\kappa V_{\mu}(x, y)+\kappa_{p} P(x, y),
\end{aligned}
$$

where $\alpha$ and $\beta$ are weighting coefficients that control, respectively, the elasticity of the contour and its rigidity, and $X^{\prime \prime}(s)$ and $X^{\prime \prime \prime}(s)$ denote the second and fourth derivatives of $X(s)$ with respect to $s . P$ is a pressure force orthogonal to the contour, and $V_{\mu}$ a GVF force that depends on a regularization parameter $\mu$ and which is nearly equal to the gradient of the edge map $f$ when it is large, but forcing the field to be slowly varying in homogenous regions. The $\kappa$ coefficient controls the GVF force and $\kappa_{p}$ the pressure force.

A first contour was defined on the filtered images using the point $P_{0}$ for the initialization and the GVF snake method with a high value of the rigidity parameter $\alpha=1, \beta=60, \kappa=1.7, \kappa_{p}=0.4$, and $\mu=0.3$. Using these parameters, this first segmentation was frequently inside the true contour, with a distance up to two or three pixels. Thus, it was used to initialize the second segmentation performed on the original image (ie, the unfiltered image) and searching between the first obtained contour and its dilation of a few pixels, in order to recover boundary information that might be lost during the filtering process. In this step, lower values of $\beta=1, \kappa_{p}=0.1$, and $\mu=0.1$ were used, while keeping $\alpha=1$ and $\kappa=1.7$ to refine the contour and to pick up small details on the endocardium.

\section{Estimation of Regional Mean Transition Times and Mean Radial Endocardial Velocities}

The contour estimated from the end-diastolic image was used to limit the analysis of parametric images to the region inside the contour. For each of the six segments, the mean transition time $T_{m c}$ was defined as the $T_{m c}$ value in the largest "isotime" region inside the segment, an "isotime" region being a set of contiguous 
pixels with the same value of $T_{m c}$. This parameter is higher for a delayed contraction (8). To allow comparison between subjects, segmental mean transition times were normalized by the RR interval duration $\left(F_{m c}=\right.$ $\left.T_{m c} / \mathrm{RR}\right)$. The combination of the parametric image $T_{O N}$ and a distance map estimated from the endocardial border depicted on the end-diastolic image resulted for each segment in a dotplot associating distances and times (Fig. 1c). The slope of the linear regression of this dotplot was defined as the mean segmental radial velocity (8).

\section{Evaluation Protocol and Statistical Analysis}

Validation of the Segmentation Procedure

An experienced cardiologist drew manual contours using the MASS software (Medis Medical Imaging Systems, Leiden, the Netherlands) for a subset of 20 studies. The area similarity $S_{\text {area }}(16)$ between the automatic and the manual segmentations was calculated. Furthermore, maximal and mean distances between the two contours (12) were estimated, as well as their standard deviations (SDs). Moreover, segmental functional parameters were estimated using both manual and automated end-diastolic endocardial contours. Results were compared using linear regression and Bland-Altman analyses.

\section{Assessment of the Range of Values for the Functional Parameters}

For the 1752 segments of the database (36 subjects, with an average of 8 slices per subject), the values of $F_{m c}$ and $V_{m}$ obtained with the automated method were analyzed according to the location in terms of slice level (AP, PM, MV), myocardial wall (A, AL, IL, I, IS, AS), and temporal resolution. Statistical tests were performed with JMP software (SAS Institute, Cary, NC), one-way and factorial analysis of variance (ANOVA), followed if necessary by protected $t$-tests, and Tukey's HSD (honestly significant difference) tests (18).

\section{RESULTS}

\section{Validation of the Segmentation Procedure}

Figure 2 shows an example of the manual and automatic segmentations where papillary muscles were included in the LV cavity. The visual observation of estimated contours was an essential control step in the estimation of the automated contours. In most cases the standard parameter values given in the Method section yielded good results. In the remaining cases (44 slices), which mostly included apical slices, these values yielded underestimated contours on the filtered images. Thus, a lower value of $\beta=50$ or higher value of $\kappa_{p}=0.6$ were used for an adequate expansion of the snake. For the second step of the segmentation, the parameters defined in the method section yielded a correct solution, except in six subjects for which the values were increased to $\beta=5$ and $\kappa_{p}=0.3$. These contours were then used for the quantification, without any manual correction.
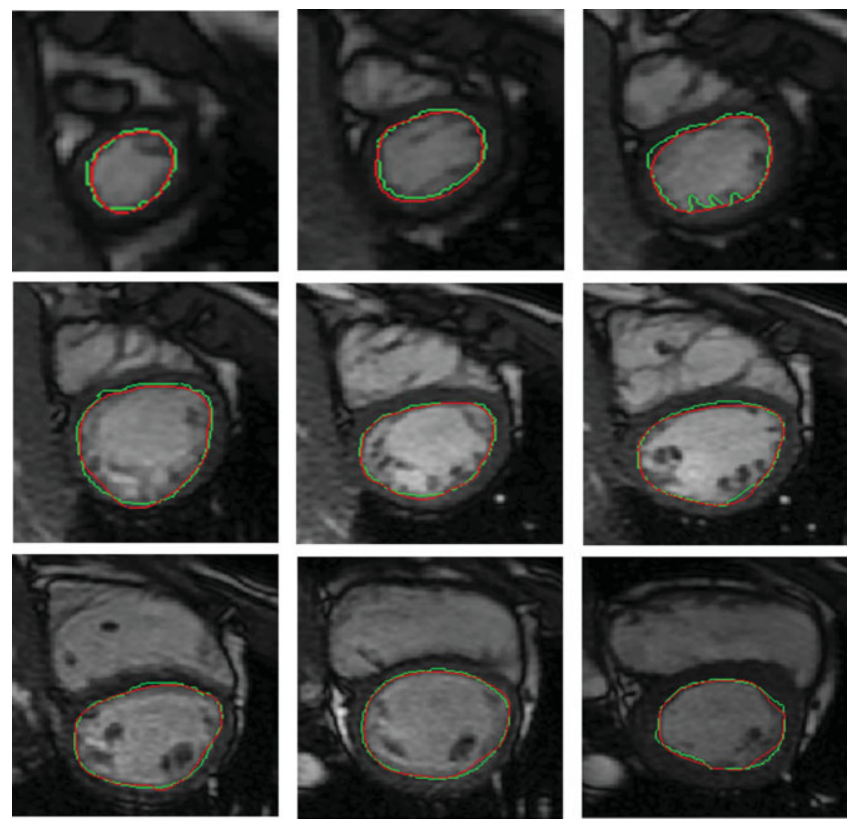

Figure 2. Results of the automated segmentation (red) versus the manual (green) segmentation for nine slice levels from the apex (top left) to the base (bottom right) in one subject.

For the 164 slices corresponding to the subset of 20 patients, the endocardial surfaces derived from the automated and the manually drawn contours were used to perform a linear regression which resulted in ( $y=$ $1.002 x+8.6, R=0.994)$, and a Bland-Altman plot that resulted in bias, upper, and lower limits of 12, 158, and $-133 \mathrm{~mm}^{2}$. The area similarity $S_{\text {area }}$ was equal to $0.91 \pm 0.04$. Values of $2.8 \pm 1.1 \mathrm{~mm}$ and $1.1 \pm 1.0 \mathrm{~mm}$ were estimated for, respectively, the maximum and mean distances between the manual and automatic contours.

In this subgroup (20 patients), the normalized mean transition times $F_{m c}$ and radial endocardial velocities $V_{m}$ were estimated using both manual and automatic contours ( $n=984$ segments). Figure 3 shows the corresponding linear regression and Bland-Altman plots.

\section{Assessment of the Range of Values for the Functional Parameters}

The $F_{m c}$ and $V_{m}$ parameters were averaged for the 1752 segments corresponding to the 36 subjects resulting, respectively, in $0.392 \pm 0.069$ and $5.4 \pm 2.3 \mathrm{~cm} / \mathrm{s}$. Figure 4 shows an example of a bull's-eye display of $F_{m c}$ and $V_{m}$ illustrating their spatial variations. ANOVA was applied to determine the combined effects of the slice level and of the myocardial wall on $F_{m c}$ values. When considering the six locations associated to myocardial walls, the comparison of $F_{m c}$ values using a protected $t$-test indicated three different groups, with values being higher in inferoseptal and anteroseptal, intermediate in inferolateral and anterolateral, and lower in inferior and anterior. Moreover, $F_{m c}$ values showed an increasing gradient from apex to base (Table 1). The coefficient of variation was higher for $V_{m}(43 \%)$ than for $F_{m c}(18 \%)$, but some significant trends could still be 

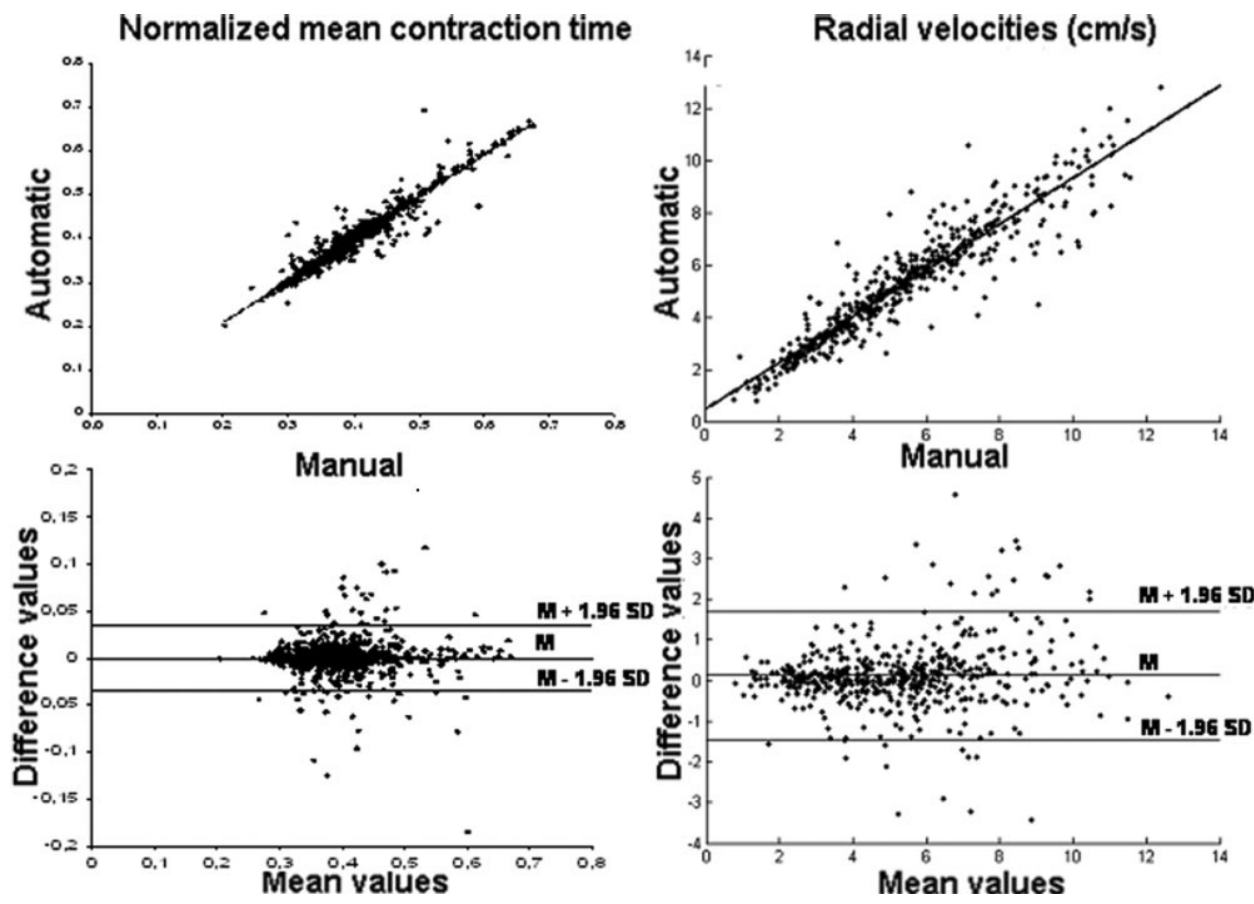

Figure 3. Comparison of functional regional parameters $(n=$ 984) estimated with both manual and automatic contours. Linear regression analysis of $F_{m c}$ and $V_{m}$ gives $(y=0.959 x+$ $0.016, R=0.964)$, and $(y=$ $0.883 x+0.505, R=0.935)$. Bias, upper, and lower values for the Bland-Altman analysis are: $0,0.035$, and -0.035 for $F_{m c}, 0.12,1.7$, and $-1.5 \mathrm{~cm} / \mathrm{s}$ for $V_{m}$. observed: inferoseptal and anteroseptal walls had a reduced velocity $(4.2$ and $3.7 \mathrm{~cm} / \mathrm{s})$ when compared to anterolateral and inferolateral walls (6.7 and $6.8 \mathrm{~cm} / \mathrm{s})$. The basal slices also had reduced velocities when compared to apical or mid-ventricular slices (Table 1).

ANOVA was performed to assess the effect of temporal resolution, slice location, and segment location on regional mean radial velocities $\left(V_{m}\right)$ and normalized mean transition times $\left(F_{m c}\right)$. This analysis indicated that both slice and segment location had a significant effect on $V_{m}$ and $F_{m c}\left(P<10^{-3}\right)$ while temporal resolution had a significant effect only on $F_{m c}(P=0.04)$.

\section{DISCUSSION}

The present study proposes a robust approach combining the detection of the endocardial border and a quantitative parametric imaging method to derive from standard cine MR images functional parameters related to time and mean radial velocities of the endocardial motion.

The LV segmentation method takes into account the presence of papillary muscles and the nonhomogeneity of the cavity using morphological filters, which successfully merged various gray level zones into the cavity. Thanks to the homogeneity of the filtered LV cavity, the initialization of the GVF snake algorithm was performed using a single point $\left(P_{O}\right)$. In addition, this homogeneity renders the resulting segmentation robust according to the location of the point $P_{0}$. Indeed, as long as $P_{O}$ was placed inside the homogeneous part of the LV cavity in the native image, the segmentation process was carried out successfully. The parameter $\lambda$ used for the filtering is sensitive to differences in LV cavity size; therefore, the choice of the optimal $\lambda$ was performed independently for each slice using an automated criterion based on the variation of the estimate of the LV cavity surface with the GVF snake according to $\lambda$ (Fig. 1). In this first step, the GVF snake algorithm provided a rough contour, which was thereafter refined using the gradient information of the original image. In addition, the GVF parameter settings were straightforward and nearly the same for all the slices. Indeed, special settings were required only for $15 \%$ of the 292 analyzed slices.
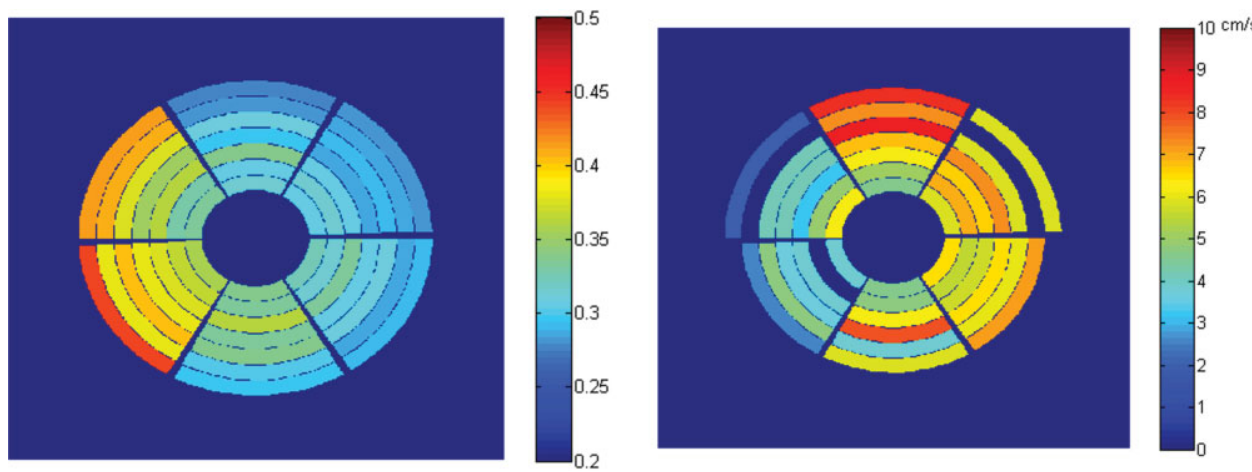

Figure 4. Bull's-eye representation of normalized mean transition times (left-hand side) and radial endocardial velocities (right-hand side) for one subject. Larger values of mean transition times are observed in inferoseptal and anteroseptal regions, especially in basal slices. Conversely, reduced values of mean radial velocities (displayed in $\mathrm{cm} / \mathrm{s}$ ) are observed in those regions. 
Table 1

Distribution of the Functional Parameters According to the Myocardial Wall and the Slice Level

\begin{tabular}{lcc}
\hline & $\begin{array}{c}\text { Normalized } \\
\text { mean transition } \\
\text { times } F_{m c}\end{array}$ & $\begin{array}{c}\text { Endocardial } \\
\text { velocities } \\
V_{m}(\mathrm{~cm} / \mathrm{s})\end{array}$ \\
\hline Anterior & $0.366 \pm 0.046$ & $5.2 \pm 1.8$ \\
Anterolateral & $0.393 \pm 0.060$ & $6.7 \pm 2.2$ \\
Inferolateral & $0.386 \pm 0.067$ & $6.8 \pm 2.3$ \\
Inferior & $0.367 \pm 0.063$ & $5.6 \pm 2.1$ \\
Inferoseptal & $0.422 \pm 0.080$ & $4.2 \pm 1.9$ \\
Anteroseptal & $0.415 \pm 0.070$ & $3.7 \pm 1.5$ \\
& & \\
Apical level & $0.375 \pm 0.057$ & $6.0 \pm 2.2$ \\
Mid-ventricular level & $0.383 \pm 0.055$ & $5.6 \pm 2.2$ \\
Basal level & $0.432 \pm 0.091$ & $4.2 \pm 2.0$ \\
\hline
\end{tabular}

To allow a comparison with other studies, the quality of the segmentation was expressed using different criteria such as comparison of endocardial areas, area similarity, and mean distances between contours. The areas provided by the automated segmentation method was in good agreement with those provided by the manual tracing as indicated by a Bland-Altman bias of 12 $\mathrm{mm}^{2}$ and a linear regression that resulted in a slope close to one and $R=0.994$; this latter was equivalent to the values reported in different studies $(9,11,14)$. Furthermore, the mean distance between the two contours was equal to $1.1 \pm 1.0 \mathrm{~mm}$ for our dataset, which is quite satisfactory when compared with $2.3 \pm 0.9 \mathrm{~mm}$ (10), $2.5 \pm 1.2 \mathrm{~mm}(12), 1.2 \pm 1.3 \mathrm{~mm}$ (13), and $1.4 \pm$ $0.5 \mathrm{~mm}(14)$.

Using this segmentation, the quantification method was automated. A comparison of the functional parameters estimated using manual and automated contours was performed using both linear regression and BlandAltman analyses (Fig. 3), which resulted in a good agreement.

Moreover, these parameters were estimated on the entire study group and both ranges for normal values and physiological differences between segments were provided and were consistent with results previously reported (8). Physiological differences in contraction times were characterized by an increase in $F_{m c}$ from apex to base, with the highest values in the septal wall as previously described (4). Mean radial endocardial velocity ranged between 3.7 and $6.8 \mathrm{~cm} / \mathrm{s}$ and were consistent with those provided by Miyatake et al (19), ie, $5.1 \pm 1.0 \mathrm{~cm} / \mathrm{s}$ in the posterior wall and slightly higher than those obtained in another echocardiography study (20) that ranged between 1.9 and $4.9 \mathrm{~cm} / \mathrm{s}$. This slight overestimation was probably due to the differences in the directions of measurements (along the ultrasound beam in echocardiography and along the perpendicular to the endocardial border in our approach). In addition, our values showed the same trend as those described in the literature with the lowest mean radial velocity values in the anteroseptal wall $(20,21)$ and a decrease from apex to base (5). Accordingly, the ability of our technique to detect small physiological delays encourages its application in the context of myocardial ischemia (22) to help in objective detection of LV dysfunction. In such applications, increased values for mean transition times and decreased velocities are expected, as previously shown on a small number of patients with myocardial infarct (8).

In this study, only subjects with normal shape were included. This is a limitation of the segmentation step because distorted LV shapes would be more difficult to segment. In most of LV distortions, especially in those where the myocardium is visible and surrounds the LV cavity, this difficulty should be minimized thanks to the filtering step, which homogenizes the LV cavity, and to the possibility to vary the parameters of the GVF snake. Also, since visual control would be necessary, the possibility to manually correct the border is available.

Another limitation is that only subjects with normal LV function were studied. Although the effect of temporal resolution on the quantitative measurements was low when compared to the effect of slice and segment location, the extension of our technique to pathological cases would require acquisitions with high temporal resolutions to be able to estimate mean radial velocities in segments with reduced motion. However, establishing segmental normal ranges of such functional parameters is a necessary step before extending the proposed approach to the detection of regional wall motion abnormalities.

Finally, similar to all techniques that track endocardial motion and/or estimate myocardial velocities, our technique may be affected by cardiac translation. In addition, the visualization of the parametric images in which the color pattern would be distorted with the presence of such motion could be helpful to discard cases with an important global translation.

In conclusion, we developed an original and robust segmentation approach and demonstrated its ability to correctly depict the endocardial border. Its combination with an automated parametric imaging of LV wall motion quantification increased its objectivity and therefore its usefulness in a clinical setting. The results of its application on a large group of normal segments demonstrated that functional parameters such as mean transition time and endocardial velocity are sensitive enough to detect normal physiological differences related to anatomical location. Therefore, these functional parameters could be used as potential markers for characterizing LV motion in the context of myocardial ischemia.

\section{REFERENCES}

1. Plein S, Bloomer T, Ridgway J, Jones T, Bainbridge G, Sivananthan $M$. Steady-state free precession magnetic resonance imaging of the heart: comparison with segmented k-space gradient-echo imaging. J Magn Reson Imaging 2001;24:593-603.

2. Hoffmann R, von Bardeleben S, Kasprzak J, et al. Analysis of regional left ventricular function by cineventriculography, cardiac magnetic resonance imaging, and unenhanced and contrast-enhanced echocardiography. A multicenter comparison of methods. J Am Coll Cardiol 2006;47:121-128.

3. Caiani E, Toledo E, MacEneaney P, Collins K, Lang R, Mor-Avi V. The role of still-frame parametric imaging in magnetic resonance assessment of left ventricular wall motion by non-cardiologists. J Cardiovasc Magn Reson 2004;6:619-625. 
4. Zwanenburg J, Gotte M, Kuijer J, Heethaar R, van Rossum A, Marcus J. Timing of cardiac contraction in humans mapped by high-temporal-resolution MRI tagging: early onset and late peak of shortening in lateral wall. Am J Physiol Heart Circ Physiol 2004; 286:1872-1880.

5. Kvitting J, Ebbers T, Engvall J, Sutherland G, Wranne B, Wigstrom L. Three-directional myocardial motion assessed using 3D phase contrast MRI. J Cardiovasc Magn Reson 2004;6:627-636.

6. Redheuil A, Kachenoura N, Laporte R, et al. Interobserver variability in assessing segmental function can be reduced by combining visual analysis of CMR cine sequences with corresponding parametric images of myocardial contraction. J Cardiovasc Magn Reson 2007;9:863-872.

7. Ruiz-Dominguez C, Kachenoura N, De Cesare A, et al. Assessment of left ventricular contraction by parametric analysis of main motion (PAMM): theory and application for echocardiography. Phys Med Biol 2005;50:3277-3296.

8. Kachenoura N, Redheuil A, Balvay D, et al. Evaluation of regional myocardial function using automated wall motion analysis of cine MR images: contribution of parametric images, contraction times, and radial velocities. J Magn Reson Imaging 2007;26:1127-1132.

9. Santarelli M, Positano V, Michelassi C, Lombardi M, Landini L. Automated cardiac MR image segmentation: theory and measurement evaluation. Med Eng Phys 2003;25:149-159.

10. Kaus M, von Berg J, Weese R, Niessen W, Pekar V. Automated segmentation of the left ventricle in cardiac MRI. Med Image Anal 2004;8:245-254.

11. Tanki N, Murase K, Kumashiro M, et al. Quantification of left ventricular volumes from cardiac cine MRI using active contour model combined with gradient vector flow. Magn Reson Med Sci 2005;4:191-196.

12. Fukami T, Sato H, Wu J, et al. Quantitative evaluation of myocardial function by a volume-normalized map generated from relative blood flow. Phys Med Biol 2007;52:4311-4330.
13. Lynch M, Ghita O, Whelan P. Segmentation of the left ventricle of the heart in 3-D+t MRI data using an optimized nonrigid temporal model. IEEE Trans Med Imaging 2008;27:195-203.

14. Andreopoulos A, Tsotsos J. Efficient and generalizable statistical models of shape and appearance for analysis of cardiac MRI. Med Image Anal 2008;12:335-357.

15. Xu C, Prince J. Snakes, shapes, and gradient vector flow. IEEE Trans Med Imaging 1998;7:359-369.

16. El Berbari R, Frouin F, Redheuil A, et al. [Development and evaluation of an automatic segmentation method of endocardial border in cardiac magnetic resonance images.] ITBM-RBM 2007;28:117123.

17. Meijster A, Wilkinson M. A comparison of algorithms for connected Set Openings and Closings. IEEE Trans Patt Anal Mach Intell 2002;24:484-494.

18. Kramer CY. Extension of multiple range tests to group means with unequal numbers of replications. Biometrics 1956;12:309-310.

19. Miyatake K, Yamagishi M, Tanaka N, et al. New method for evaluating left ventricular wall motion by color-coded tissue Doppler imaging: in vitro and in vivo studies. J Am Coll Cardiol 1995;25: 717-724.

20. Wilkenshoff UM, Sovany A, Wigström L, et al. Regional mean systolic myocardial velocity estimation by real-time color Doppler myocardial imaging: a new technique for quantifying regional systolic function. J Am Soc Echocardiogr 1998;11:683-9221.

21. Hennig J, Schneider B, Peschl S, Michael M, Krause T, Laubenberger J. Analysis of myocardial motion based on velocity measurements with a black blood prepared segmented gradient-echo sequence: methodology and applications to normal volunteers and patients. J Magn Reson Imaging 1998;8:868-877.

22. Lucats L, Monnet X, Bize A, et al. Regional and temporal heterogeneity of postsystolic wall thickening is associated with left ventricular asynchrony in normal and experimental stunned myocardium. Basic Res Cardiol 2008;103:385-396. 